\title{
COMBINED MONTE CARLO SAMPLING AND PENALTY METHOD FOR STOCHASTIC NONLINEAR COMPLEMENTARITY PROBLEMS
}

\author{
GUI-HUA LIN
}

\begin{abstract}
In this paper, we consider a new formulation with recourse for a class of stochastic nonlinear complementarity problems. We show that the new formulation is equivalent to a smooth semi-infinite program that no longer contains recourse variables. We then propose a combined Monte Carlo sampling and penalty method for solving the problem in which the underlying sample space is assumed to be compact. Furthermore, we suggest a compact approximation approach for the case where the sample space is unbounded. Two preliminary numerical examples are included as well.
\end{abstract}

\section{INTRODUCTION}

The nonlinear complementarity problem (NCP) is one of the fundamental problems in the optimization theory and its applications can be found in many fields. See the monographs [9] and [10] for details. Since in many practical problems, some elements may involve uncertain data, stochastic versions of NCP (called SNCP below) have been receiving more and more attention in the recent literature [6, 7, 14, 30, 11, 12, 17. In this paper, we consider the following stochastic nonlinear complementarity problem: Find a vector $x$ such that

$$
\mathcal{P}\{\omega \in \Omega \mid 0 \leq x \perp F(x, \omega) \geq 0\}=1,
$$

where $(\Omega, \Xi, \mathcal{P})$ is the underlying probability space, $F: \Re^{n} \times \Omega \rightarrow \Re^{n}$ is a given function, and the symbol $\perp$ means the two vectors are perpendicular to each other. This problem can be rewritten as

$$
0 \leq x \perp F(x, \omega) \geq 0, \quad \omega \in \Omega \text { a.s. },
$$

where "a.s." is the abbreviation for "almost surely" under the given probability measure. Throughout, we suppose that $F(x, \omega)$ is continuously differentiable with respect to $x$.

Note that problem (1.1) may not have a solution in general. There have been proposed three ways to deal with (1.1): One way is suggested by Gürkan et al. [12, who use an expectation instead to give a single nonlinear complementarity problem. Another way is presented by Chen and Fukushima [6], who make use of the so-called NCP functions to present the expected residual minimization formulation for (1.1).

Received by the editor May 14, 2007 and, in revised form, January 26, 2008 and July 13, 2008. 2000 Mathematics Subject Classification. Primary 90C33; Secondary 90C30, 90C15.

Key words and phrases. Stochastic nonlinear complementarity problem, recourse, Monte Carlo method, penalization, convergence.

This work was supported in part by NSFC Grant \#10771025 and SRFDP Grant \#20070141063.

(c)2009 American Mathematical Society Reverts to public domain 28 years from publication 1671 
See [7, 14, 30, 11] for recent developments along this way. The last is proposed by Lin and Fukushima [17, who formulate (1.1) as the following model:

$$
\begin{array}{cl}
\min & \mathbb{E}\left[d^{T} z(\omega)\right] \\
\text { s.t. } & 0 \leq x \perp(F(x, \omega)+z(\omega)) \geq 0, \\
& z(\omega) \geq 0, \quad \omega \in \Omega \text { a.s., }
\end{array}
$$

where $\mathbb{E}$ means expectation with respect to the random variable $\omega \in \Omega, z(\omega)$ is a recourse variable, and $d \in \Re^{n}$ is a constant vector with positive elements. A smoothing penalty method based on some reformulations of (1.2) has been proposed in 17 for the case where $\Omega$ has finite elements. In this paper, we continue to study the last way and focus on dealing with a general case where $\Omega$ is a subset of $\Re^{l}$.

Note that problem (1.2) is actually a stochastic mathematical program with equilibrium constraints (SMPEC), which plays an important role in many fields such as engineering design, economic equilibrium, and multilevel games. See 20, 1, 15, 16, 18, 19, 21, 22, 26, 27, 28, 29] for details. Problem (1.2) has the following main difficulties:

- The problem contains an expectation and a recourse variable depending on $\omega$. Both of them may cause computational difficulty in general.

- Because of the presence of complementarity constraints, problem (1.2) fails to satisfy a standard constraint qualification at any feasible point 8 .

Based on these observations, our first purpose is to eliminate the recourse variable. To this end, we propose the following formulation of SNCP, which slightly differs from (1.2):

$$
\begin{array}{cl}
\min & \mathbb{E}\left[\|z(\omega)\|^{2}\right] \\
\text { s.t. } & 0 \leq x \perp(F(x, \omega)+z(\omega)) \geq 0, \\
& z(\omega) \geq 0, \omega \in \Omega \text { a.s. }
\end{array}
$$

The reasons we consider (1.3) instead of (1.2) can be stated as follows:

- Since both $d^{T} z(\omega)$ and $\|z(\omega)\|^{2}$ serve as penalty terms for the possible violation of the complementarity constraint $0 \leq x \perp F(x, \omega) \geq 0$, problems (1.2) and (1.3) are essentially the same.

- The quadratic penalty $\|z(\omega)\|^{2}$ yields an equivalent problem

$$
\begin{array}{cl}
\min & \mathbb{E}\left[\|u(x, \omega)\|^{2}\right] \\
\text { s.t. } & x \geq 0, \\
& x \circ F(x, \omega) \leq 0, \omega \in \Omega \text { a.s., }
\end{array}
$$

which has a differentiable objective function, but the linear penalty $d^{T} z(\omega)$ yields an equivalent problem

$$
\begin{array}{cl}
\min & \mathbb{E}\left[d^{T} u(x, \omega)\right] \\
\text { s.t. } & x \geq 0, \\
& x \circ F(x, \omega) \leq 0, \quad \omega \in \Omega \text { a.s., }
\end{array}
$$

whose objective function is not differentiable everywhere. Here, $u: \Re^{n} \times$ $\Omega \rightarrow \Re^{n}$ is a function defined by

$$
u(x, \omega):=\max \{-F(x, \omega), 0\}
$$


and $\circ$ denotes the Hadamard product, i.e.,

$$
x \circ F(x, \omega):=\left(x_{1} F_{1}(x, \omega), \ldots, x_{n} F_{n}(x, \omega)\right)^{T} .
$$

See Section 2 for a proof of the equivalence between (1.3) and (1.4). Note that problem (1.4) no longer contains the recourse variable.

As known to us, the sample average approximation (SAA) approach is wellknown and most popular in the literature on SMPEC, and it has been extensively studied 21, 22, 27, 28, 19. In general, SAA methods for SMPEC always require some techniques to deal with the variational inequality or complementarity constraints. For example,

- for the case where the variational inequality or complementarity constraints take on a form of the expectations of the functions involved, Lin et al. 19] utilize some merit function to rewrite the constraints as nonsmooth equations and then employ a smoothing and penalty technique to get some approximation problems, whereas Meng and $\mathrm{Xu}$ [22] make use of regularization or smoothing technique to handle the constraints;

- for the case where the variational inequality or complementarity constraints are not described by expectations, a popular way is to apply some implicit function theorems as well as some techniques including regularization or smoothing technique; see for instance Meng and Xu [21] and Shapiro and $\mathrm{Xu}$ [27, 28].

Thanks to an effort to remove the recourse variable, problem (1.2) has been successfully reformulated to (1.4), which does not contain complementarity constraints. However, problem (1.4) is actually a semi-infinite programming problem with a large number of complementarity-like constraints and it also involves an expectation in the objective function. Therefore, problem (1.4) is generally more difficult to handle than an ordinary semi-infinite programming problem. In this paper, as in the works [21, 22, 27, 28, 19, we will make use of a Monte Carlo sampling method to handle the expectation and employ a penalty technique to deal with the complementarity-like constraints. Throughout, we denote by $\mathcal{F}$ the feasible region of problem (1.4). It is clear that $\mathcal{F}$ is nonempty.

\section{Equivalence Between (1.3) AND (1.4)}

We show the equivalence between problems (1.3) and (1.4) below.

Theorem 2.1. If $x^{*}$ solves (1.4), then $\left(x^{*}, u\left(x^{*}, \cdot\right)\right)$ is an optimal solution of problem (1.3). Conversely, if $\left(x^{*}, z^{*}(\cdot)\right)$ is an optimal solution of problem (1.3), then $x^{*}$ solves (1.4).

Proof. (i) Suppose that $x^{*}$ is an optimal solution of (1.4). We then have from (1.5) that

$$
F\left(x^{*}, \omega\right)+u\left(x^{*}, \omega\right) \geq 0, \quad \forall \omega \in \Omega .
$$

Note that, if $x_{i}^{*}>0$ for some $i$, there must hold $F_{i}\left(x^{*}, \omega\right) \leq 0$ for almost all $\omega \in \Omega$ and so $u_{i}\left(x^{*}, \omega\right)=-F_{i}\left(x^{*}, \omega\right)$ for almost all $\omega \in \Omega$. Therefore, we have

$$
\left(x^{*}\right)^{T}\left(F\left(x^{*}, \omega\right)+u\left(x^{*}, \omega\right)\right)=0, \quad \omega \in \Omega \text { a.s. }
$$

This indicates that $\left(x^{*}, u\left(x^{*}, \cdot\right)\right)$ is feasible to problem (1.3). Let $(x, z(\cdot))$ be an arbitrary feasible point of problem (1.3). It then follows that, for almost every 
$\omega \in \Omega$,

$$
z(\omega)-u(x, \omega)=\min \{F(x, \omega)+z(\omega), z(\omega)\} \geq 0
$$

and hence $z(\omega) \geq u(x, \omega) \geq 0$. This implies that

$$
\mathbb{E}\left[\|z(\omega)\|^{2}-\|u(x, \omega)\|^{2}\right] \geq 0 .
$$

On the other hand, it follows from the feasibility of $(x, z(\cdot))$ in (1.3) that

$$
x \circ F(x, \omega)=-x \circ z(\omega) \leq 0, \quad \omega \in \Omega \text { a.s. }
$$

and so $x$ is a feasible point of (1.4). Thus, we have from the optimality of $x^{*}$ in (1.4) that

$$
\mathbb{E}\left[\|u(x, \omega)\|^{2}\right] \geq \mathbb{E}\left[\left\|u\left(x^{*}, \omega\right)\right\|^{2}\right] .
$$

It follows from (2.1) and (2.2) that

$$
\begin{aligned}
& \mathbb{E}\left[\|z(\omega)\|^{2}\right]-\mathbb{E}\left[\left\|u\left(x^{*}, \omega\right)\right\|^{2}\right] \\
& =\mathbb{E}\left[\|u(x, \omega)\|^{2}\right]-\mathbb{E}\left[\left\|u\left(x^{*}, \omega\right)\right\|^{2}\right]+\mathbb{E}\left[\|z(\omega)\|^{2}-\|u(x, \omega)\|^{2}\right] \\
& \geq 0 .
\end{aligned}
$$

This indicates that $\left(x^{*}, u\left(x^{*}, \cdot\right)\right)$ is an optimal solution of problem (1.3).

(ii) Suppose that $\left(x^{*}, z^{*}(\cdot)\right)$ is an optimal solution of (1.3). It is not difficult to see that $z^{*}(\omega)=u\left(x^{*}, \omega\right)$ for almost all $\omega \in \Omega$. Let $x$ be an arbitrary feasible point of (1.4). In a similar way to (i), we can show that $(x, u(x, \cdot))$ is feasible to problem (1.3). Since $\left(x^{*}, z^{*}(\cdot)\right)$ solves (1.3), there holds

$$
\mathbb{E}\left[\|u(x, \omega)\|^{2}\right] \geq \mathbb{E}\left[\left\|z^{*}(\omega)\right\|^{2}\right]=\mathbb{E}\left[\left\|u\left(x^{*}, \omega\right)\right\|^{2}\right] .
$$

This implies that $x^{*}$ is an optimal solution of problem (1.4).

\section{Boundedness OF LEVEL SETS}

In this section, we discuss conditions for boundedness of the level sets of the objective function in problem (1.4). For simplicity, we denote by

$$
\begin{aligned}
& f(x):=\mathbb{E}[F(x, \omega)], \\
& \theta(x):=\mathbb{E}\left[\|u(x, \omega)\|^{2}\right],
\end{aligned}
$$

and consider the level set defined by

$$
L_{\mathcal{F}}^{\theta}(c):=\{x \in \mathcal{F} \mid \theta(x) \leq c\},
$$

where $c \geq 0$ is a given scalar. Recall that $f$ is an $R_{0}$-function on $\mathcal{F}$ if for any sequence $\left\{x^{k}\right\} \subseteq \mathcal{F}$ satisfying

$$
\begin{aligned}
& \lim _{k \rightarrow \infty}\left\|x^{k}\right\|=+\infty, \\
& \limsup _{k \rightarrow \infty}\left\|\min \left\{x^{k}, 0\right\}\right\|<+\infty, \\
& \limsup _{k \rightarrow \infty}\left\|\min \left\{f\left(x^{k}\right), 0\right\}\right\|<+\infty,
\end{aligned}
$$

there exists an index $j$ such that $x_{j}^{k} \rightarrow+\infty$ and $f_{j}\left(x^{k}\right) \rightarrow+\infty$ as $k \rightarrow+\infty$. This property is a generation of the so-called $R_{0}$ matrix, whose definition will be given in the next section, and it relates closely to boundedness of level sets in complementarity theory. See [4, 5, 10] for more details. 
Theorem 3.1. Assume that $f$ is an $R_{0}$-function on $\mathcal{F}$. Then, for any nonnegative scalar $c$, the level set $L_{\mathcal{F}}^{\theta}(c)$ is bounded.

Proof. Suppose that there is a nonnegative number $\bar{c}$ such that the level set $L_{\mathcal{F}}^{\theta}(\bar{c})$ is unbounded. This implies that there exists a sequence $\left\{x^{k}\right\} \subseteq L_{\mathcal{F}}^{\theta}(\bar{c})$ such that $\lim _{k \rightarrow \infty}\left\|x^{k}\right\|=+\infty$. It is obvious that, for each $k$,

$$
\begin{aligned}
\bar{c} & \geq \theta\left(x^{k}\right) \\
& =\sum_{i=1}^{n} \mathbb{E}\left[u_{i}^{2}\left(x^{k}, \omega\right)\right] \\
& \geq \sum_{i=1}^{n}\left(\mathbb{E}\left[u_{i}\left(x^{k}, \omega\right)\right]\right)^{2} \\
& =\sum_{i=1}^{n}\left(\mathbb{E}\left[\max \left\{-F_{i}\left(x^{k}, \omega\right), 0\right\}\right]\right)^{2} \\
& \geq \sum_{i=1}^{n} \max ^{2}\left\{-f_{i}\left(x^{k}\right), 0\right\},
\end{aligned}
$$

where the last two inequalities follow from Jensen's inequality. From (3.2), we have

$$
f_{i}\left(x^{k}\right) \geq-\sqrt{\bar{c}}, \quad \forall i, \forall k .
$$

Note that $x^{k} \geq 0$ for every $k$. This together with (3.3) indicates that condition (3.1) is satisfied. Since $f$ is an $R_{0}$-function on $\mathcal{F}$, there exists an index $j$ such that

$$
\lim _{k \rightarrow \infty} x_{j}^{k}=+\infty, \quad \lim _{k \rightarrow \infty} f_{j}\left(x^{k}\right)=+\infty .
$$

On the other hand, for each $k$, by the feasibility of $x^{k}$ in problem (1.4), we have

$$
x^{k} \circ F\left(x^{k}, \omega\right) \leq 0, \quad \omega \in \Omega \text { a.s. }
$$

and so $x^{k} \circ f\left(x^{k}\right) \leq 0$ for each $k$. This obviously contradicts (3.4) and hence the proof is complete.

Since $\mathcal{F}$ is nonempty, we see from Theorem 3.1 that problem (1.4) has at least one optimal solution when $f$ is an $R_{0}$-function on $\mathcal{F}$.

\section{Monte Carlo sampling and penalty approximations}

Let $\Omega$ be a compact set and $\phi: \Omega \rightarrow \Re$ an integrable function. The Monte Carlo sampling estimate for $\mathbb{E}[\phi(\omega)]$ is obtained by taking independently and identically distributed random samples $\Omega_{k}:=\left\{\omega_{1}, \ldots, \omega_{N_{k}}\right\}$ from $\Omega$ and letting $\mathbb{E}[\phi(\omega)] \approx$ $\frac{1}{N_{k}} \sum_{\omega_{\ell} \in \Omega_{k}} \phi\left(\omega_{\ell}\right)$. The strong law of large numbers guarantees that this procedure converges with probability one (abbreviated by "w.p.1"below), i.e.,

$$
\lim _{k \rightarrow \infty} \frac{1}{N_{k}} \sum_{\omega_{\ell} \in \Omega_{k}} \phi\left(\omega_{\ell}\right)=\mathbb{E}[\phi(\omega)]:=\int_{\Omega} \phi(\omega) d p \quad \text { w.p.1. }
$$

See 23, 25 for more details about the Monte Carlo sampling methods. 
Applying the above method and using a penalty technique, we obtain the problem

$$
\begin{array}{ll}
\min & \frac{1}{N_{k}} \sum_{\omega_{\ell} \in \Omega_{k}}\left(\left\|u\left(x, \omega_{\ell}\right)\right\|^{2}+\rho_{k}\left\|x \circ v\left(x, \omega_{\ell}\right)\right\|^{2}\right) \\
\text { s.t. } & x \geq 0,
\end{array}
$$

which is a smooth approximation of problem (1.4). Here, $\rho_{k}>0$ is a penalty parameter tending to $\infty$ as $k \rightarrow \infty, u: \Re^{n} \times \Omega \rightarrow \Re^{n}$ is defined by (1.5), and $v: \Re^{n} \times \Omega \rightarrow \Re^{n}$ is given by

$$
v(x, \omega):=\max \{F(x, \omega), 0\} .
$$

Note that, by (1.5) and (4.3), we have

$$
v(x, \omega)=F(x, \omega)+u(x, \omega), \quad(x, \omega) \in \Re^{n} \times \Omega .
$$

Problem (4.2) is neither a semi-infinite program nor an MPEC and it is generally much easier to deal with than those problems. Note that the feasible region of problem (4.2) does not depend on $k$.

4.1. Existence of solutions. We next discuss the existence conditions of solutions of problem (4.2). For simplicity, we assume that $F$ is affine with respect to $x$ and given by

$$
F(x, \omega):=M(\omega) x+q(\omega),
$$

where $M: \Omega \rightarrow \Re^{n \times n}$ and $q: \Omega \rightarrow \Re^{n}$ are continuous.

Definition 4.1. We call $\bar{M} \in \Re^{n \times n}$ an $R_{0}$-matrix if

$$
x \geq 0, \bar{M} x \geq 0, x^{T} \bar{M} x=0 \quad \Longrightarrow \quad x=0 .
$$

There are a number of matrix classes having been given in the monograph 9 . These matrix classes play important roles in the theory of the linear complementarity problems. In particular, $R_{0}$-matrices are often employed in existence theory of solutions and convergence analysis of algorithms. It is well known that the classes, which at least include positive definite matrix class, $P$-matrix class, and strictly copositive matrix class, belong to the $R_{0}$-matrix class 9 . In addition, as mentioned in Section 3, these properties have been extended to nonlinear cases; see 4, 5, 10, for details.

Lemma 4.2. Let $\left\{M_{k}\right\} \subset \Re^{n \times n}$ be convergent to $\bar{M} \in \Re^{n \times n}$ and let $\bar{M}$ be an $R_{0}$-matrix. Then, there exists an integer $k_{0}>0$ such that $M_{k}$ is an $R_{0}$-matrix for every $k \geq k_{0}$.

This lemma is easy to verify and so we omit its proof here.

Theorem 4.3. Let $\Omega_{k}=\left\{\omega_{1}, \ldots, \omega_{N_{k}}\right\}$ be a set of independently and identically distributed random samples drawn from $\Omega$ for each $k$ and $\rho_{k}$ tend to $+\infty$ as $k \rightarrow \infty$. Suppose that $\frac{1}{N_{k}} \sum_{\omega_{\ell} \in \Omega_{k}} M\left(\omega_{\ell}\right)$ converges to an $R_{0}$-matrix $\bar{M}$. We then have the following statements almost surely:

(i) Problem (4.2) has at least one optimal solution when $k$ is sufficiently large.

(ii) Let $x^{k}$ be an optimal solution of (4.2) for each $k$ sufficiently large. Then the sequence $\left\{x^{k}\right\}$ is bounded. 
Proof. (i) For each $k$, let $M_{k}:=\frac{1}{N_{k}} \sum_{\omega_{\ell} \in \Omega_{k}} M\left(\omega_{\ell}\right)$. It follows that $\bar{M}=\lim _{k \rightarrow \infty} M_{k}$. Since $\bar{M}$ is an $R_{0}$-matrix, by Lemma 4.2, there exists an integer $k_{0}>0$ such that $M_{k}$ is an $R_{0}$-matrix for every $k \geq k_{0}$.

Let $k \geq k_{0}$ be fixed. Note that the objective function of problem (4.2) is bounded below on $\Re_{+}^{n}$. Then, there exists a sequence $\left\{x^{j}\right\} \subseteq \Re_{+}^{n}$ such that

$$
\begin{aligned}
& \lim _{j \rightarrow \infty} \frac{1}{N_{k}} \sum_{\omega_{\ell} \in \Omega_{k}}\left(\left\|u\left(x^{j}, \omega_{\ell}\right)\right\|^{2}+\rho_{k}\left\|x^{j} \circ v\left(x^{j}, \omega_{\ell}\right)\right\|^{2}\right) \\
& =\inf _{x \geq 0} \frac{1}{N_{k}} \sum_{\omega_{\ell} \in \Omega_{k}}\left(\left\|u\left(x, \omega_{\ell}\right)\right\|^{2}+\rho_{k}\left\|x \circ v\left(x, \omega_{\ell}\right)\right\|^{2}\right) .
\end{aligned}
$$

Since $\rho_{k}$ is a positive constant, it follows from (4.6) that the sequences

$$
\left\{\frac{1}{N_{k}} \sum_{\omega_{\ell} \in \Omega_{k}}\left\|u\left(x^{j}, \omega_{\ell}\right)\right\|^{2}\right\}_{j=0,1, \ldots}
$$

and

$$
\left\{\frac{1}{N_{k}} \sum_{\omega_{\ell} \in \Omega_{k}}\left\|x^{j} \circ v\left(x^{j}, \omega_{\ell}\right)\right\|^{2}\right\}_{j=0,1, \ldots}
$$

are bounded. This along with (4.4) implies that

$$
\left\{\frac{1}{N_{k}} \sum_{\omega_{\ell} \in \Omega_{k}} u\left(x^{j}, \omega_{\ell}\right)\right\}_{j=0,1, \ldots}
$$

and

$$
\left\{\frac{1}{N_{k}} \sum_{\omega_{\ell} \in \Omega_{k}}\left(x^{j}\right)^{T}\left(M\left(\omega_{\ell}\right) x^{j}+q\left(\omega_{\ell}\right)+u\left(x^{j}, \omega_{\ell}\right)\right)\right\}_{j=0,1, \ldots}
$$

are also bounded. Note that the latter sequence can be rewritten as

$$
\left\{\left(x^{j}\right)^{T}\left(M_{k} x^{j}+\frac{1}{N_{k}} \sum_{\omega_{\ell} \in \Omega_{k}} q\left(\omega_{\ell}\right)+\frac{1}{N_{k}} \sum_{\omega_{\ell} \in \Omega_{k}} u\left(x^{j}, \omega_{\ell}\right)\right)\right\}_{j=0,1, \ldots} .
$$

On the other hand, it is obvious from the definition of $u$ that, for each $j$,

$$
x^{j} \geq 0, \quad M_{k} x^{j}+\frac{1}{N_{k}} \sum_{\omega_{\ell} \in \Omega_{k}} q\left(\omega_{\ell}\right)+\frac{1}{N_{k}} \sum_{\omega_{\ell} \in \Omega_{k}} u\left(x^{j}, \omega_{\ell}\right) \geq 0 .
$$

Suppose the sequence $\left\{x^{j}\right\}$ is unbounded. Taking a subsequence if necessary, we assume that

$$
\lim _{j \rightarrow \infty}\left\|x^{j}\right\|=+\infty, \quad \lim _{j \rightarrow \infty} \frac{x^{j}}{\left\|x^{j}\right\|}=\bar{x}, \quad\|\bar{x}\|=1 .
$$

Then, dividing (4.7) and (4.8) by $\left\|x^{j}\right\|^{2}$ and $\left\|x^{j}\right\|$, respectively, and letting $j \rightarrow+\infty$, we obtain

$$
0 \leq \bar{x} \perp M_{k} \bar{x} \geq 0 .
$$

Since $M_{k}$ is an $R_{0}$-matrix, we have $\bar{x}=0$. This contradicts (4.9) and hence $\left\{x^{j}\right\}$ is bounded.

Therefore, $\left\{x^{j}\right\}$ is bounded. Since $\Re_{+}^{n}$ is closed, we see from (4.6) that any accumulation point of $\left\{x^{j}\right\}$ must be an optimal solution of (4.2). This completes the proof of (i). 
(ii) Let $x^{k}$ be an optimal solution of (4.2) for each sufficiently large $k$. We next prove that $\left\{x^{k}\right\}$ is almost surely bounded. Since $x^{k}$ is an optimal solution of (4.2), we have

$$
\frac{1}{N_{k}} \sum_{\omega_{\ell} \in \Omega_{k}}\left(\left\|u\left(x^{k}, \omega_{\ell}\right)\right\|^{2}+\rho_{k}\left\|x^{k} \circ v\left(x^{k}, \omega_{\ell}\right)\right\|^{2}\right) \leq \frac{1}{N_{k}} \sum_{\omega_{\ell} \in \Omega_{k}}\left\|u\left(0, \omega_{\ell}\right)\right\|^{2}
$$

and, by the definitions (1.5) and (4.5),

(4.11) $\frac{1}{N_{k}} \sum_{\omega_{\ell} \in \Omega_{k}} M\left(\omega_{\ell}\right) x^{k}+\frac{1}{N_{k}} \sum_{\omega_{\ell} \in \Omega_{k}} q\left(\omega_{\ell}\right)+\frac{1}{N_{k}} \sum_{\omega_{\ell} \in \Omega_{k}} u\left(x^{k}, \omega_{\ell}\right) \geq 0, \quad x^{k} \geq 0$.

Noting that $\mathbb{E}[F(0, \omega)]<+\infty$ by the continuity of $F$ and the compactness of $\Omega$, we have from (4.1) that $\left\{\frac{1}{N_{k}} \sum_{\omega_{\ell} \in \Omega_{k}}\left\|u\left(0, \omega_{\ell}\right)\right\|^{2}\right\}$ is almost surely bounded. So, the sequences

$$
\left\{\frac{1}{N_{k}} \sum_{\omega_{\ell} \in \Omega_{k}}\left\|u\left(x^{k}, \omega_{\ell}\right)\right\|^{2}\right\} \quad \text { and } \quad\left\{\frac{\rho_{k}}{N_{k}} \sum_{\omega_{\ell} \in \Omega_{k}}\left\|x^{k} \circ v\left(x^{k}, \omega_{\ell}\right)\right\|^{2}\right\}
$$

are almost surely bounded. By Cauchy-Schwarz inequality, we have

$$
\left(\sum_{\omega_{\ell} \in \Omega_{k}} u_{i}\left(x^{k}, \omega_{\ell}\right)\right)^{2} \leq N_{k} \sum_{\omega_{\ell} \in \Omega_{k}}\left(u_{i}\left(x^{k}, \omega_{\ell}\right)\right)^{2}, \quad i=1, \ldots, n
$$

for each $k$ and hence

$$
\begin{aligned}
\left\|\frac{1}{N_{k}} \sum_{\omega_{\ell} \in \Omega_{k}} u\left(x^{k}, \omega_{\ell}\right)\right\|^{2} & =\frac{1}{N_{k}^{2}} \sum_{i=1}^{n}\left(\sum_{\omega_{\ell} \in \Omega_{k}} u_{i}\left(x^{k}, \omega_{\ell}\right)\right)^{2} \\
& \leq \frac{1}{N_{k}} \sum_{i=1}^{n} \sum_{\omega_{\ell} \in \Omega_{k}}\left(u_{i}\left(x^{k}, \omega_{\ell}\right)\right)^{2} \\
& =\frac{1}{N_{k}} \sum_{\omega_{\ell} \in \Omega_{k}}\left\|u\left(x^{k}, \omega_{\ell}\right)\right\|^{2} .
\end{aligned}
$$

Similarly, we have

$$
\begin{aligned}
& \left|\frac{1}{N_{k}} \sum_{\omega_{\ell} \in \Omega_{k}}\left(x^{k}\right)^{T}\left(M\left(\omega_{\ell}\right) x^{k}+q\left(\omega_{\ell}\right)+u\left(x^{k}, \omega_{\ell}\right)\right)\right|^{2} \\
& =\frac{1}{N_{k}^{2}}\left|\sum_{i=1}^{n} \sum_{\omega_{\ell} \in \Omega_{k}} x_{i}^{k} v_{i}\left(x^{k}, \omega_{\ell}\right)\right|^{2} \\
& \leq \frac{n}{N_{k}^{2}} \sum_{i=1}^{n}\left(\sum_{\omega_{\ell} \in \Omega_{k}} x_{i}^{k} v_{i}\left(x^{k}, \omega_{\ell}\right)\right)^{2} \\
& \leq \frac{n}{N_{k}} \sum_{i=1}^{n} \sum_{\omega_{\ell} \in \Omega_{k}}\left(x_{i}^{k} v_{i}\left(x^{k}, \omega_{\ell}\right)\right)^{2} \\
& =\frac{n}{N_{k}} \sum_{\omega_{\ell} \in \Omega_{k}}\left\|x^{k} \circ v\left(x^{k}, \omega_{\ell}\right)\right\|^{2} .
\end{aligned}
$$

It follows from (4.12) and (4.13) that both $\left\{\frac{1}{N_{k}} \sum_{\omega_{\ell} \in \Omega_{k}} u\left(x^{k}, \omega_{\ell}\right)\right\}$ and

$$
\left\{\frac{1}{N_{k}} \sum_{\omega_{\ell} \in \Omega_{k}}\left(x^{k}\right)^{T}\left(M\left(\omega_{\ell}\right) x^{k}+q\left(\omega_{\ell}\right)+u\left(x^{k}, \omega_{\ell}\right)\right)\right\}
$$


are almost surely bounded. Suppose that the sequence $\left\{x^{k}\right\}$ is unbounded with probability $\pi>0$. Taking a subsequence if necessary, we assume that

$$
\lim _{k \rightarrow \infty}\left\|x^{k}\right\|=+\infty, \quad \lim _{k \rightarrow \infty} \frac{x^{k}}{\left\|x^{k}\right\|}=\bar{x}, \quad\|\bar{x}\|=1
$$

hold with positive probability $\pi$. Note that $\left\{x^{k}\right\}$ and $\left\{\frac{1}{N_{k}} \sum_{\omega_{\ell} \in \Omega_{k}} u\left(x^{k}, \omega_{\ell}\right)\right\}$ are almost surely bounded and, by (4.1),

$$
\lim _{k \rightarrow \infty} \frac{1}{N_{k}} \sum_{\omega_{\ell} \in \Omega_{k}} M\left(\omega_{\ell}\right)=\bar{M}, \quad \lim _{k \rightarrow \infty} \frac{1}{N_{k}} \sum_{\omega_{\ell} \in \Omega_{k}} q\left(\omega_{\ell}\right)=\int_{\Omega} q(\omega) d p
$$

with probability one. Dividing (4.11) and (4.14) by $\left\|x^{k}\right\|$ and $\left\|x^{k}\right\|^{2}$, respectively, and letting $k \rightarrow+\infty$, we have $0 \leq \bar{x} \perp \bar{M} \bar{x} \geq 0$ with positive probability $\pi$. Since $\bar{M}$ is an $R_{0}$-matrix, we have $\bar{x}=0$ with positive probability $\pi$. This contradicts (4.15) and hence, the sequence $\left\{x^{k}\right\}$ is almost surely bounded. This completes the proof of (ii).

4.2. Convergence analysis. In this subsection, we investigate convergence properties of the Monte Carlo sampling and penalty method. For each $k$, we let $\left\{\omega_{1}, \ldots, \omega_{N_{k}}\right\}$ be independently and identically distributed random samples drawn from $\Omega$.

Definition 4.4 ([24]). Let $\tau>0$ and $\kappa \geq 0$ be constants. We say $G: \Re^{n} \rightarrow \Re^{m}$ is Hölder continuous on $K \subseteq \Re^{n}$ with order $\tau$ and Hölder constant $\kappa$ if

$$
\|G(x)-G(y)\| \leq \kappa\|x-y\|^{\tau}
$$

holds for all $x$ and $y$ in $K$.

This concept is a generalization of the Lipschitz continuity, which is, by definition, Hölder continuity with order $\tau=1$. Note that, for two different positive numbers $\tau$ and $\tau^{\prime}$, Hölder continuous functions with order $\tau$ and those with order $\tau^{\prime}$ constitute different subclasses. For example, the function $G(x):=\sqrt{\|x\|}$ is Hölder continuous with order $\tau=\frac{1}{2}$ but not Lipschitz continuous. See [24] for more details on Hölder continuity.

Theorem 4.5. Suppose that $F$ is Hölder continuous in $x$ on $\Re_{+}^{n}$ with order $\tau>0$ and Hölder constant $\kappa(\omega)$ satisfying $\mathbb{E}[\kappa(\omega)]<+\infty$. Assume that $\lim _{k \rightarrow \infty} \rho_{k}=+\infty$, $x^{k}$ solves problem (4.2) for each $k$, and $x^{*}$ is an accumulation point of $\left\{x^{k}\right\}$. Then $x^{*}$ is an optimal solution of problem (1.4) with probability one.

Proof. Without loss of generality, we suppose that $\lim _{k \rightarrow \infty} x^{k}=x^{*}$.

(i) We first prove that $x^{*}$ is almost surely feasible to (1.4). It is obvious that $x^{*} \geq 0$. Therefore, it is sufficient to show

$$
x^{*} \circ F\left(x^{*}, \omega\right) \leq 0, \quad \omega \in \Omega \text { a.s. }
$$


In fact, since $x^{k}$ is an optimal solution of problem (4.2), we have

$$
\begin{aligned}
& \frac{\rho_{k}}{N_{k}} \sum_{\omega_{\ell} \in \Omega_{k}}\left\|x^{k} \circ v\left(x^{k}, \omega_{\ell}\right)\right\|^{2} \\
& \leq \frac{1}{N_{k}} \sum_{\omega_{\ell} \in \Omega_{k}}\left(\left\|u\left(x^{k}, \omega_{\ell}\right)\right\|^{2}+\rho_{k}\left\|x^{k} \circ v\left(x^{k}, \omega_{\ell}\right)\right\|^{2}\right) \\
& \leq \frac{1}{N_{k}} \sum_{\omega_{\ell} \in \Omega_{k}}\left\|u\left(0, \omega_{\ell}\right)\right\|^{2} .
\end{aligned}
$$

Since $\Omega$ is compact, the function $u(0, \cdot)$ is bounded on $\Omega$. Thus, we have from (4.17) that $\left\{\frac{\rho_{k}}{N_{k}} \sum_{\omega_{\ell} \in \Omega_{k}}\left\|x^{k} \circ v\left(x^{k}, \omega_{\ell}\right)\right\|^{2}\right\}$ is bounded. As a result,

$$
\left\{\frac{\rho_{k}}{N_{k}} \sum_{\omega_{\ell} \in \Omega_{k}}\left(x_{i}^{k}\right)^{2}\left(F_{i}\left(x^{k}, \omega_{\ell}\right)+u_{i}\left(x^{k}, \omega_{\ell}\right)\right)^{2}\right\}
$$

is bounded for each $i$ and, since

$$
x^{k} \geq 0, \quad F\left(x^{k}, \omega_{\ell}\right)+u\left(x^{k}, \omega_{\ell}\right)=v\left(x^{k}, \omega_{\ell}\right) \geq 0
$$

for every $k$ and $\ell,\left\{\frac{\rho_{k}}{N_{k}} \sum_{\omega_{\ell} \in \Omega_{k}}\left(x^{k}\right)^{T}\left(F\left(x^{k}, \omega_{\ell}\right)+u\left(x^{k}, \omega_{\ell}\right)\right)\right\}$ is bounded. Noting that $\lim _{k \rightarrow \infty} \rho_{k}=+\infty$, we have

$$
\lim _{k \rightarrow \infty} \frac{1}{N_{k}} \sum_{\omega_{\ell} \in \Omega_{k}}\left(x^{k}\right)^{T}\left(F\left(x^{k}, \omega_{\ell}\right)+u\left(x^{k}, \omega_{\ell}\right)\right)=0 .
$$

On the other hand, by the assumptions of the theorem, there holds

$$
\left\|F(x, \omega)-F\left(x^{\prime}, \omega\right)\right\| \leq \kappa(\omega)\left\|x-x^{\prime}\right\|^{\tau}
$$

for any $x, x^{\prime} \in \Re_{+}^{n}$ and $\omega \in \Omega$. It follows that, for any $k$ and $\ell$,

$$
\begin{aligned}
\left\|\left(F\left(x^{k}, \omega_{\ell}\right)+u\left(x^{k}, \omega_{\ell}\right)\right)-\left(F\left(x^{*}, \omega_{\ell}\right)+u\left(x^{*}, \omega_{\ell}\right)\right)\right\| & \leq 2\left\|F\left(x^{k}, \omega_{\ell}\right)-F\left(x^{*}, \omega_{\ell}\right)\right\| \\
& \leq 2 \kappa\left(\omega_{\ell}\right)\left\|x^{k}-x^{*}\right\|^{\tau}
\end{aligned}
$$

and then

$$
\begin{aligned}
& \lim _{k \rightarrow \infty}\left|\frac{1}{N_{k}} \sum_{\omega_{\ell} \in \Omega_{k}}\left(x^{k}\right)^{T}\left(\left(F\left(x^{k}, \omega_{\ell}\right)+u\left(x^{k}, \omega_{\ell}\right)\right)-\left(F\left(x^{*}, \omega_{\ell}\right)+u\left(x^{*}, \omega_{\ell}\right)\right)\right)\right| \\
& \leq \lim _{k \rightarrow \infty} 2\left\|x^{k}\right\|\left\|x^{k}-x^{*}\right\|^{\tau} \frac{1}{N_{k}} \sum_{\omega_{\ell} \in \Omega_{k}} \kappa\left(\omega_{\ell}\right) \\
& =0 \quad \text { w.p.1. }
\end{aligned}
$$

We have from (4.18) and (4.20) that

$$
\begin{aligned}
0 & =\lim _{k \rightarrow \infty} \frac{1}{N_{k}} \sum_{\omega_{\ell} \in \Omega_{k}}\left(x^{k}\right)^{T}\left(F\left(x^{k}, \omega_{\ell}\right)+u\left(x^{k}, \omega_{\ell}\right)\right) \\
& =\lim _{k \rightarrow \infty} \frac{1}{N_{k}} \sum_{\omega_{\ell} \in \Omega_{k}}\left(x^{k}\right)^{T}\left(F\left(x^{*}, \omega_{\ell}\right)+u\left(x^{*}, \omega_{\ell}\right)\right) \\
& =\int_{\Omega}\left(x^{*}\right)^{T}\left(F\left(x^{*}, \omega\right)+u\left(x^{*}, \omega_{\ell}\right)\right) d p \quad \text { w.p. } 1,
\end{aligned}
$$

where the last equality follows from (4.1). Noting that both $\left(x^{*}\right)^{T}\left(F\left(x^{*}, \cdot\right)+u\left(x^{*}, \cdot\right)\right)$ and $\left(x^{*}\right)^{T} u\left(x^{*}, \cdot\right)$ are nonnegative on $\Omega$, we obtain (4.16) from (4.21) immediately. 
(ii) Let $x$ be an arbitrary feasible solution of (1.4). If $x_{i}>0$ for some $i$, then $F_{i}(x, \omega) \leq 0$ holds for almost all $\omega \in \Omega$ and so $u_{i}(x, \omega)=-F_{i}(x, \omega)$ for almost all $\omega \in \Omega$. This means

$$
x \circ v(x, \omega)=x \circ(F(x, \omega)+u(x, \omega))=0, \quad \omega \in \Omega \text { a.s. }
$$

Since $x^{k}$ is an optimal solution of problem (4.2), we have almost surely that

$$
\begin{aligned}
\frac{1}{N_{k}} \sum_{\omega_{\ell} \in \Omega_{k}}\left\|u\left(x, \omega_{\ell}\right)\right\|^{2} & =\frac{1}{N_{k}} \sum_{\omega_{\ell} \in \Omega_{k}}\left(\left\|u\left(x, \omega_{\ell}\right)\right\|^{2}+\rho_{k}\left\|x \circ v\left(x, \omega_{\ell}\right)\right\|^{2}\right) \\
& \geq \frac{1}{N_{k}} \sum_{\omega_{\ell} \in \Omega_{k}}\left(\left\|u\left(x^{k}, \omega_{\ell}\right)\right\|^{2}+\rho_{k}\left\|x^{k} \circ v\left(x^{k}, \omega_{\ell}\right)\right\|^{2}\right) \\
& \geq \frac{1}{N_{k}} \sum_{\omega_{\ell} \in \Omega_{k}}\left\|u\left(x^{k}, \omega_{\ell}\right)\right\|^{2} .
\end{aligned}
$$

As a result, we have

$$
\begin{aligned}
& \frac{1}{N_{k}} \sum_{\omega_{\ell} \in \Omega_{k}}\left\|u\left(x^{*}, \omega_{\ell}\right)\right\|^{2}-\frac{1}{N_{k}} \sum_{\omega_{\ell} \in \Omega_{k}}\left\|u\left(x, \omega_{\ell}\right)\right\|^{2} \\
& \leq \frac{1}{N_{k}} \sum_{\omega_{\ell} \in \Omega_{k}}\left\|u\left(x^{*}, \omega_{\ell}\right)\right\|^{2}-\frac{1}{N_{k}} \sum_{\omega_{\ell} \in \Omega_{k}}\left\|u\left(x^{k}, \omega_{\ell}\right)\right\|^{2} \\
& \leq \frac{1}{N_{k}} \sum_{\omega_{\ell} \in \Omega_{k}}\left\|u\left(x^{*}, \omega_{\ell}\right)-u\left(x^{k}, \omega_{\ell}\right)\right\|\left(\left\|u\left(x^{*}, \omega_{\ell}\right)\right\|+\left\|u\left(x^{k}, \omega_{\ell}\right)\right\|\right) \quad \text { w.p.1. }
\end{aligned}
$$

It follows from (1.5) and (4.19) that

$$
\begin{aligned}
\left\|u\left(x^{*}, \omega_{\ell}\right)-u\left(x^{k}, \omega_{\ell}\right)\right\| & \leq\left\|F\left(x^{*}, \omega_{\ell}\right)-F\left(x^{k}, \omega_{\ell}\right)\right\| \\
& \leq \kappa\left(\omega_{\ell}\right)\left\|x^{k}-x^{*}\right\|^{\tau}, \quad \ell=1, \ldots, N_{k} .
\end{aligned}
$$

By the boundedness of the sequence $\left\{\frac{1}{N_{k}} \sum_{\omega_{\ell} \in \Omega_{k}}\left(\left\|u\left(x^{*}, \omega_{\ell}\right)\right\|+\left\|u\left(x^{k}, \omega_{\ell}\right)\right\|\right)\right\}$, we have

$$
\lim _{k \rightarrow \infty} \frac{1}{N_{k}} \sum_{\omega_{\ell} \in \Omega_{k}}\left\|u\left(x^{*}, \omega_{\ell}\right)-u\left(x^{k}, \omega_{\ell}\right)\right\|\left(\left\|u\left(x^{*}, \omega_{\ell}\right)\right\|+\left\|u\left(x^{k}, \omega_{\ell}\right)\right\|\right)=0 .
$$

Letting $k \rightarrow+\infty$ in (4.23) and taking (4.24) and (4.1) into account, we obtain

$$
\mathbb{E}\left[\left\|u\left(x^{*}, \omega\right)\right\|^{2}\right] \leq \mathbb{E}\left[\|u(x, \omega)\|^{2}\right] \quad \text { w.p. } 1,
$$

which indicates that $x^{*}$ is an optimal solution of (1.4) with probability one.

Remark 4.6. In a recent publication [2], Bastin et al. also studied the Monte Carlo methods for stochastic programming. Their purpose was to investigate the limiting behavior of stationary points of the approximation problems. Some convergence results based on first-order or second-order conditions are given. Compared with this paper, the original stochastic programming of 2 has a deterministic and compact feasible region and so it is different from (1.4) which has a random feasible region. Moreover, the convergence results given in [2] require a uniformly convergence assumption of the mappings including the objective functions, constraint functions, and their gradients. See 2 for details. 
4.3. Numerical examples. We have tested the proposed method on the following examples.

Example 4.7. Consider the stochastic complementarity problem (1.1) in which $\omega$ is uniformly distributed on $\Omega:=[0,1]$ and $F: \Re^{3} \times \Omega \rightarrow \Re^{3}$ is given by

$$
F(x, \omega):=\left(\begin{array}{c}
x_{1}-\omega x_{2}+3-2 \omega \\
-\omega x_{1}+2 x_{2}+\omega x_{3}-2-\omega \\
\omega x_{2}+3 x_{3}-3-\omega
\end{array}\right) .
$$

This problem has a unique solution $x^{*}=(0,1,1)$ for each $\omega \in \Omega$. Therefore, the optimal value of (1.3) corresponding to this example is zero, as shown in Table 1

Example 4.8. Consider the stochastic complementarity problem (1.1) in which $\omega$ is uniformly distributed on $\Omega:=[0,1]$ and $F: \Re^{2} \times \Omega \rightarrow \Re^{2}$ is given by

$$
F(x, \omega):=\left(\begin{array}{c}
x_{1}+\omega x_{2}-2+\omega \\
\omega x_{1}+2 x_{2}+1+\omega
\end{array}\right) .
$$

This problem has no common solution for all $\omega \in \Omega$. Note that (1.3) becomes

$$
\begin{array}{ll}
\min & \mathbb{E}\left[\|z(\omega)\|^{2}\right] \\
\text { s.t. } & 0 \leq\left(\begin{array}{l}
x_{1} \\
x_{2}
\end{array}\right) \perp\left(\begin{array}{c}
x_{1}+\omega x_{2}-2+\omega \\
\omega x_{1}+2 x_{2}+1+\omega
\end{array}\right)+z(\omega) \geq 0, \\
& z(\omega) \geq 0, \omega \in[0,1] .
\end{array}
$$

Let $\left(x_{1}, x_{2}, z(\cdot)\right)$ be an arbitrary feasible point of (4.25). Since $\omega x_{1}+2 x_{2}+1+\omega>0$ for $\omega \in[0,1]$, we have $x_{2}=0$. Let $z^{*}(\omega):=\left(\begin{array}{c}2-\omega-x_{1} \\ 0\end{array}\right)$. Note that, if $x_{1} \neq 0$, then $x_{1}-2+\omega+z_{1}(\omega)=0$ holds. It follows that $\left(x_{1}, x_{2}, z^{*}(\cdot)\right)$ is feasible to problem (4.25) and $z(\omega) \geq z^{*}(\omega) \geq 0$ for any $\omega \in[0,1]$. Furthermore, we have

$$
\mathbb{E}\left[\|z(\omega)\|^{2}\right] \geq \mathbb{E}\left[\left\|z^{*}(\omega)\right\|^{2}\right]=\int_{0}^{1}\left(2-\omega-x_{1}\right)^{2} d \omega=x_{1}^{2}-3 x_{1}+\frac{7}{3} .
$$

Recall that $x_{1} \leq 2-\omega$ for any $\omega \in[0,1]$. It follows that $x_{1} \in[0,1]$. Thus, we get an optimal solution $\left(1,0, z^{*}(\cdot)\right)$ of (4.25) with $z^{*}(\omega):=\left(\begin{array}{c}1-\omega \\ 0\end{array}\right)$. It is easy to see that the optimal value of problem (4.25) is $\frac{1}{3}$.

Since the functions given in Examples 4.7 and 4.8 have the form of (4.5), they are evidently Lipschitz continuous in $x$ with Lipschitz constant $\kappa(\omega)=\|M(\omega)\|$. Here $\|M(\omega)\|$ denotes the Euclidean operator norm of $M(\omega)$. By straightforward calculations, we have

- for Example 4.7

$$
\mathbb{E}[M(\omega)]=\left(\begin{array}{ccc}
1 & -1 / 2 & 0 \\
-1 / 2 & 2 & 1 / 2 \\
0 & 1 / 2 & 3
\end{array}\right)
$$

is an $R_{0}$ matrix and $\mathbb{E}[\kappa(\omega)]=\mathbb{E}\left[2+\sqrt{1+2 \omega^{2}}\right]<+\infty$;

- for Example 4.8 .

$$
\mathbb{E}[M(\omega)]=\left(\begin{array}{cc}
1 & 1 / 2 \\
1 / 2 & 2
\end{array}\right)
$$

is an $R_{0}$ matrix and $\mathbb{E}[\kappa(\omega)]=\mathbb{E}\left[\frac{3+\sqrt{1+4 \omega^{2}}}{2}\right]<+\infty$.

This means that the assumptions made in Theorems 4.3 and 4.5 are satisfied. 
Table 1. Computational Results for Examples 4.74 .8

\begin{tabular}{|c|c|c|c|c|}
\hline \multirow{2}{*}{ Parameters } & \multicolumn{2}{|c|}{ Example 4.7} & \multicolumn{2}{c|}{ Example 4.8} \\
\cline { 2 - 5 } & $x^{k}$ & Obj & $x^{k}$ & Obj \\
\hline$N_{1}=10^{2}, \rho_{1}=10^{2}$ & $(0,1.0019,0.9489)$ & $6.8918 \mathrm{e}-004$ & $(1.1153,0)$ & 0.7905 \\
\hline$N_{2}=10^{3}, \rho_{2}=10^{3}$ & $(0,0.9996,0.9982)$ & $6.8476 \mathrm{e}-006$ & $(1.0329,0)$ & 0.4175 \\
\hline$N_{3}=10^{4}, \rho_{3}=10^{4}$ & $(0,0.9999,0.9985)$ & $1.3156 \mathrm{e}-006$ & $(1.0027,0)$ & 0.3242 \\
\hline$N_{4}=10^{5}, \rho_{4}=10^{5}$ & $(0,1.0000,0.9995)$ & $9.9033 \mathrm{e}-008$ & $(1.0007,0)$ & 0.3301 \\
\hline
\end{tabular}

In our experiments, we set the initial values of $N_{k}$ and $\rho_{k}$ as $N_{1}:=10^{2}$ and $\rho_{1}:=10^{2}$, respectively. Then, we employed the random number generator rand in Matlab 6.5 to generate independently and identically distributed random samples $\left\{\omega_{1}, \ldots, \omega_{N_{k}}\right\}$ from $\Omega$ and we solved the subproblems

$$
\min _{x \geq 0} \frac{1}{N_{k}} \sum_{\omega_{\ell} \in \Omega_{k}}\left(\left\|\max \left(-F\left(x, \omega_{\ell}\right), 0\right)\right\|^{2}+\rho_{k}\left\|x \circ \max \left(F\left(x, \omega_{\ell}\right), 0\right)\right\|^{2}\right)
$$

by the solver fmincon in Matlab 6.5 to get a point $x^{k}$. The initial point was chosen to be $(0, \ldots, 0)$ and the computed solution $x^{k}$ was used as the starting point in the next iteration. In addition, the parameters were updated by $N_{k}:=10 N_{k}$ and $\rho_{k}:=\min \left\{10 \rho_{k}, \bar{\rho}\right\}$ with $\bar{\rho}=10^{5}$. The computational results for Examples 4.7 and 4.8 are shown in Table 1, in which Obj denotes the values of the objective function of (4.26) at the current point. The results shown in the table reveal that the proposed method was able to solve the examples successfully.

\section{THE CASE WHERE $\Omega$ IS UNBOUNDED}

In the last section, the sample space $\Omega$ is simply assumed to be compact. In this section, we discuss the case where $\Omega$ is an unbounded and closed subset of $\Re^{l}$. For this case, one popular way is to consider compact approximations of (1.4). That is, given a sufficiently large number $\nu$, we define a compact approximation of $\Omega$ by

$$
\Omega_{\nu}:=\{\omega \in \Omega \mid\|\omega\| \leq \nu\}
$$

and consider the following approximation problem of (1.4):

$$
\begin{array}{ll}
\min & \theta_{\nu}(x):=\int_{\Omega_{\nu}}\|u(x, \omega)\|^{2} \rho(\omega) d \omega \\
\text { s.t. } & x \geq 0, \\
& x \circ F(x, \omega) \leq 0, \quad \omega \in \Omega_{\nu} \text { a.s. }
\end{array}
$$

Since problem (5.1) has a compact sample space, we may employ the method proposed in Section 4 to solve (5.1). Further convergence results are stated as follows.

Theorem 5.1. Suppose that $F$ is Hölder continuous in $x$ on $\mathcal{F}$ with order $\tau>0$ and Hölder constant $\kappa(\omega)$ satisfying $\mathbb{E}\left[\kappa^{2}(\omega)\right]<+\infty$. Suppose that there exists a vector $\hat{x}$ in $\mathcal{F}$ such that $\mathbb{E}\left[\|F(\hat{x}, \omega)\|^{2}\right]<+\infty$. Let $x^{\nu}$ be an optimal solution of problem (5.1) for each $\nu$ and let $\bar{x}$ be an accumulation point of $\left\{x^{\nu}\right\}$. Then $\bar{x}$ is an optimal solution of problem (1.4). 
Proof. Without loss of generality, we assume that $\lim _{\nu \rightarrow \infty} x^{\nu}=\bar{x}$. It is obvious that $\bar{x} \in \mathcal{F}$. Since both $\mathbb{E}\left[\kappa^{2}(\omega)\right]$ and $\mathbb{E}\left[\|F(\hat{x}, \omega)\|^{2}\right]$ are finite, it follows from CauchySchwarz inequality that $\mathbb{E}[\kappa(\omega)\|F(\hat{x}, \omega)\|]$ is also finite. Thus, by the Hölder continuity of $F$ in $x$, we have

$$
\begin{aligned}
& \mathbb{E}\left[\|F(\bar{x}, \omega)\|^{2}\right] \\
& \leq \mathbb{E}\left[(\|F(\hat{x}, \omega)\|+\|F(\bar{x}, \omega)-F(\hat{x}, \omega)\|)^{2}\right] \\
& \leq \mathbb{E}\left[\left(\|F(\hat{x}, \omega)\|+\kappa(\omega)\|\bar{x}-\hat{x}\|^{\tau}\right)^{2}\right] \\
& \leq \mathbb{E}\left[\|F(\hat{x}, \omega)\|^{2}\right]+2\|\bar{x}-\hat{x}\|^{\tau} \mathbb{E}[\kappa(\omega)\|F(\hat{x}, \omega)\|]+\|\bar{x}-\hat{x}\|^{2 \tau} \mathbb{E}\left[\kappa^{2}(\omega)\right] \\
& <+\infty
\end{aligned}
$$

and, using Cauchy-Schwarz inequality again,

$$
\mathbb{E}[\kappa(\omega)\|F(\bar{x}, \omega)\|]<+\infty .
$$

(i) We show

$$
\lim _{\nu \rightarrow \infty} \theta_{\nu}\left(x^{\nu}\right)=\theta(\bar{x}),
$$

where $\theta$ is the same as in Section 3. On the one hand, we have from (5.2) that

$$
\begin{aligned}
\left|\theta_{\nu}(\bar{x})-\theta(\bar{x})\right| & =\int_{\Omega \backslash \Omega_{\nu}}\|u(\bar{x}, \omega)\|^{2} \rho(\omega) d \omega \\
& \leq \int_{\Omega \backslash \Omega_{\nu}}\|F(\bar{x}, \omega)\|^{2} \rho(\omega) d \omega \\
& \rightarrow 0 \quad \text { as } \nu \rightarrow+\infty .
\end{aligned}
$$

On the other hand, we have from the Hölder continuity of $F$, the fact that $\mathbb{E}\left[\kappa^{2}(\omega)\right]$ is finite, and (5.3) that

$$
\begin{aligned}
& \left|\theta_{\nu}\left(x^{\nu}\right)-\theta_{\nu}(\bar{x})\right| \\
& =\left|\int_{\Omega_{\nu}}\left(\left\|u\left(x^{\nu}, \omega\right)\right\|^{2}-\|u(\bar{x}, \omega)\|^{2}\right) \rho(\omega) d \omega\right| \\
& \leq \int_{\Omega_{\nu}}\left(\left\|u\left(x^{\nu}, \omega\right)\right\|+\|u(\bar{x}, \omega)\|\right)\left\|u\left(x^{\nu}, \omega\right)-u(\bar{x}, \omega)\right\| \rho(\omega) d \omega \\
& \leq \int_{\Omega_{\nu}}\left(\left\|u\left(x^{\nu}, \omega\right)-u(\bar{x}, \omega)\right\|^{2}+2\|u(\bar{x}, \omega)\|\left\|u\left(x^{\nu}, \omega\right)-u(\bar{x}, \omega)\right\|\right) \rho(\omega) d \omega \\
& \leq \int_{\Omega_{\nu}}\left(\left\|F\left(x^{\nu}, \omega\right)-F(\bar{x}, \omega)\right\|^{2}+2\|F(\bar{x}, \omega)\|\left\|F\left(x^{\nu}, \omega\right)-F(\bar{x}, \omega)\right\|\right) \rho(\omega) d \omega \\
& \leq\left\|x^{\nu}-\bar{x}\right\|^{2 \tau} \int_{\Omega_{\nu}} \kappa^{2}(\omega) \rho(\omega) d \omega+2\left\|x^{\nu}-\bar{x}\right\|^{\tau} \int_{\Omega_{\nu}} \kappa(\omega)\|F(\bar{x}, \omega)\| \rho(\omega) d \omega \\
& \leq\left\|x^{\nu}-\bar{x}\right\|^{2 \tau} \mathbb{E}\left[\kappa^{2}(\omega)\right]+2\left\|x^{\nu}-\bar{x}\right\|^{\tau} \mathbb{E}[\kappa(\omega)\|F(\bar{x}, \omega)\|] \\
& \rightarrow 0 \quad \text { as } \nu \rightarrow+\infty .
\end{aligned}
$$

By (5.5) -(5.6) and

$$
\left|\theta_{\nu}\left(x^{\nu}\right)-\theta(\bar{x})\right| \leq\left|\theta_{\nu}\left(x^{\nu}\right)-\theta_{\nu}(\bar{x})\right|+\left|\theta_{\nu}(\bar{x})-\theta(\bar{x})\right|,
$$

we get (5.4) immediately. 
(ii) Note that $x^{\nu}$ is an optimal solution of problem (5.1) for each $\nu$ and any $x \in \mathcal{F}$ must be feasible to (5.1). Therefore, we have

$$
\theta_{\nu}\left(x^{\nu}\right) \leq \theta_{\nu}(x) \leq \theta(x), \quad \forall x \in \mathcal{F} .
$$

Letting $\nu \rightarrow+\infty$ in (5.7) and taking (5.4) into account, we obtain

$$
\theta(\bar{x}) \leq \theta(x), \quad \forall x \in \mathcal{F} .
$$

This indicates that $\bar{x}$ is an optimal solution of (1.4).

\section{Conclusions}

We have presented a new formulation (1.3) for SNCP and shown that the new formulation is actually equivalent to a smooth semi-infinite programming problem (1.4). Then, we employed a Monte Carlo sampling method and a penalty technique to get some approximations of (1.4) with compact sample space. We have also presented a compact approximation approach for the case where the sample space is unbounded.

On the other hand, recall that the sample space $\Omega$ is assumed to have infinitely many elements in the paper. Actually, if $\Omega$ has only a finite number of elements, we may present a similar method without resorting to a Monte Carlo sampling approximation technique.

\section{ACKNOWLEDGEMENTS}

The author would like to sincerely thank Professor Masao Fukushima and the anonymous referees whose helpful suggestions led to much improvement of this paper.

\section{REFERENCES}

1. S.I. Birbil, G. Gürkan, and O. Listes, Solving stochastic mathematical programs with complementarity constraints using simulation, Mathematics of Operations Research, 31 (2006), 739-760. MR.2281227 (2007k:90073)

2. F. Bastin, C. Cirillo and P.L. Toint, Convergence theory for nonconvex stochastic programming with an application to mixed logit, Mathematical Programming, 108 (2006), 207-234. MR 2238700 (2007c:90061)

3. J.R. Birge and F. Louveaux, Introduction to Stochastic Programming, Springer, New York, 1997. MR 1460264 (99b:90001)

4. B. Chen, Error bounds for $R_{0}$-type and monotone nonlinear complementarity problems, Journal of Optimization Theory and Applications, 108 (2001), 297-316. MR 1824294 (2001m:90107)

5. B. Chen and P.T. Harker, Smooth approximations to nonlinear complementarity problems, SIAM Journal on Optimization, 7 (1997), 403-420. MR.1443626 (98e:90192)

6. X. Chen and M. Fukushima, Expected residual minimization method for stochastic linear complementarity problems, Mathematics of Operations Research, 30 (2005), 1022-1038 MR2185828 (2006h:90069)

7. X. Chen, C. Zhang and M. Fukushima, Robust solution of monotone stochastic linear complementarity problems, Mathematical Programming, 117 (2009), 51-80.

8. Y. Chen and M. Florian, The nonlinear bilevel programming problem: Formulations, regularity and optimality conditions, Optimization, 32 (1995), 193-209. MR1336341 (96c:90100)

9. R.W. Cottle, J.S. Pang, and R.E. Stone, The Linear Complementarity Problem, Academic Press, New York, 1992. MR:1150683 (93f:90001)

10. F. Facchinei and J.S. Pang, Finite-Dimensional Variational Inequalities and Complementarity Problems, Springer-Verlag, New York, 2003.

11. H. Fang, X. Chen and M. Fukushima, Stochastic $R_{0}$ matrix linear complementarity problems, SIAM Journal on Optimization, 18 (2007), 482-506. MR2338448(2008f:90112) 
12. G. Gürkan, A.Y. Özge and S.M. Robinson, Sample-path solution of stochastic variational inequalities, Mathematical Programming, 84 (1999), 313-333. MR.1690005 (2000b:90094)

13. R. Hettich and K.O. Kortanek, Semi-infinite programming: Theory, methods, and applications, SIAM Review, 35 (1993), 380-429. MR1234637 (94g:90152)

14. G.H. Lin, X. Chen and M. Fukushima, New restricted NCP function and their applications to stochastic NCP and stochastic MPEC, Optimization, 56 (2007), 641-753. MR2362712 (2008g:90068)

15. G.H. Lin, X. Chen and M. Fukushima, Solving stochastic mathematical programs with equilibrium constraints via approximation and smoothing implicit programming with penalization, Mathematical Programming, 116 (2009), 343-368.

16. G.H. Lin and M. Fukushima, A class of stochastic mathematical programs with complementarity constraints: Reformulations and algorithms, Journal of Industrial and Management Optimization, 1 (2005), 99-122. MR2127807 (2006d:90111)

17. G.H. Lin and M. Fukushima, New reformulations for stochastic complementarity problems, Optimization Methods and Software, 21 (2006), 551-564. MR2277498 (2007j:90036)

18. G.H. Lin and M. Fukushima, Regularization method for stochastic mathematical programs with complementarity constraints, European Series of Applied and Industrial Mathematics (ESAIM): Control, Optimisation and Calculus of Variations, 11 (2005), 252-265. MR2141889 (2005m:90073)

19. G.H. Lin, H. Xu and M. Fukushima, Monte Carlo and quasi-Monte Carlo sampling methods for a class of stochastic mathematical programs with equilibrium constraints, Mathematical Methods of Operations Research, 67 (2008), 423-441. MR2403716

20. Z.Q. Luo, J.S. Pang, and D. Ralph, Mathematical Programs with Equilibrium Constraints, Cambridge University Press, Cambridge, United Kingdom, 1996. MR1419501 (97j:90002)

21. F. Meng and $\mathrm{H}$. Xu, A regularized sample average approximation method for stochastic mathematical programs with nonsmooth equality constraints, SIAM Journal on Optimization, 17 (2006), 891-919. MR 2257215 (2007i:90051)

22. F. Meng and H. Xu, Exponential convergence of sample average approximation methods for a class of stochastic mathematical programs with complementarity constraints, Journal of Computational Mathematics, 24 (2006), 733-748. MR2269956 (2007g:90068)

23. H. Niederreiter, Random Number Generation and Quasi-Monte Carlo Methods, SIAM, Philadelphia, 1992. MR1172997 (93h:65008)

24. J.M. Ortega and W.C. Rheinboldt, Iterative Solution of Nonlinear Equations in Several Variables, Academic Press, New York, 1970. MR0273810 (42:8686)

25. A. Shapiro, Monte Carlo sampling approach to stochastic programming, European Series of Applied and Industrial Mathematics (ESAIM): Proceedings, 13 (2003), 65-73. MR2160881 (2006d:90116)

26. A. Shapiro, Stochastic programming with equilibrium constraints, Journal of Optimization Theory and Applications, 128 (2006), 223-243. MR2201897 (2006j:90054)

27. A. Shapiro and H. Xu, Stochastic mathematical programs with equilibrium constraints, modeling and sample average approximation, Optimization, 57 (2008), 395-418. MR2412074

28. H. Xu, An implicit programming approach for a class of stochastic mathematical programs with linear complementarity constraints, SIAM Journal on Optimization, 16 (2006), 670-696. MR 2197552 (2006k:90086)

29. H. Xu and F. Meng, Convergence analysis of sample average approximation methods for a class of stochastic mathematical programs with equality constraints, Mathematics of Operations Research, 32 (2007), 648-668. MR2348240 (2008h:90068)

30. C. Zhang and X. Chen, Stochastic nonlinear complementarity problem and applications to traffic equilibrium under uncertainty, Journal of Optimization Theory and Applications, 137 (2008), 277-295. MR2395102

Department of Applied Mathematics, Dalian University of Technology, Dalian 116024, CHINA

E-mail address: lin_g_h@yahoo.com.cn 